\title{
Du climat au changement climatique : chantiers, leçons et défis pour l'histoire
}

From climate to climate change: works in progress, lessons and challenges for history

Jean-François Mouhot

\section{(2) OpenEdition Journals}

\section{Édition électronique}

URL : http://journals.openedition.org/conflits/18571

DOI : 10.4000/conflits.18571

ISSN : $1777-5345$

Éditeur :

CCLS - Centre d'études sur les conflits lilberté et sécurité, L'Harmattan

Édition imprimée

Date de publication : 30 décembre 2012

Pagination : $19-42$

ISBN : 978-2-343-00589-8

ISSN : 1157-996X

Référence électronique

Jean-François Mouhot, "Du climat au changement climatique : chantiers, leçons et défis pour

I'histoire », Cultures \& Conflits [En ligne], 88 | hiver 2012, mis en ligne le 15 mars 2014, consulté le 30 mars 2021. URL : http://journals.openedition.org/conflits/18571 ; DOI : https://doi.org/10.4000/ conflits. 18571 


\section{Du climat au changement climatique : chantiers, leçons et défis pour l'histoire*}

\section{Jean-François MOUHOT}

Jean-François Moubot est docteur en histoire et Marie Curie fellow, Georgetown University et Kluge Center, Library of Congress. Il a récemment publié : Des esclaves énergétiques. Réflexions sur le changement climatique, Champ Vallon, 2011 (Prix AARHSE 2012); Les Réfugiés acadiens en France. L’impossible réintégration ? (1758-1785), Presses universitaires de Rennes, 2012 ; (avec CharlesFrançois Mathis), Une protection de l'environnement à la française ? XIX ${ }^{\mathrm{e}}-\mathrm{XX}^{\mathrm{e}}$ siècle, Champ Vallon, 2013.

\section{Histoire et climat : champs d'investigation}

$\mathrm{D}$ ans son ouvrage classique sur l'histoire des perceptions de la nature depuis l'Antiquité jusqu'à la fin du XVIIIe siècle, Clarence Glacken nous apprend que les hommes se sont interrogés depuis fort longtemps sur leur relation à l'environnement selon trois perspectives : la terre a-t-elle été créée intentionnellement par un ou plusieurs dieux ? Quelle influence le climat, le relief, ou la configuration des continents ont-ils exercé sur les hommes et les sociétés ? Comment l'homme a-t-il transformé son cadre de vie ? 1

À l'exception de la première, ces questions sont les mêmes que celles posées par l'histoire de l'environnement. Ce nouveau champ de recherche né à la fin des années 1960 aux États-Unis - et dont l'ambition est de remettre en question certains présupposés de la discipline historique - s'intéresse en effet à la manière dont les humains ont modifié leur environnement, à l'influence de l'environnement sur les sociétés, et enfin à la façon dont les hommes ont perçu, compris ou interprété leur environnement. Ces trois questions sont

* Je voudrais remercier Olivier Godard, Franz Mauelshagen et Chloé Vlassopoulos ainsi que deux relecteurs anonymes pour leurs commentaires et suggestions. Les recherches pour cet article ont été financées en partie par le conseil européen de la recherche, International Outgoing Marie Curie fellowship n 252936.Toutes les citations de ce texte ont été traduites de l'anglais par mes soins.

1. Glacken C. J., Traces on the Rhodian shore, Berkeley, University of California Press, 1967. 
aujourd'hui posées par certains chercheurs -se définissant le plus souvent, mais pas toujours, comme historiens de l'environnement - plus spécifiquement à propos du climat.

Le réchauffement climatique global actuel suscite des recherches historiques conduites dans des champs assez différents. Le premier à s’être développé est la climatologie historique, dont le principal objectif est la reconstruction du temps et du climat avant l'apparition des instruments de mesure modernes. Les recherches historiques sur le climat ont débuté avant même que le changement climatique ne soit clairement identifié et compris comme un problème majeur. L'ouvrage d'Emmanuel Le Roy Ladurie, Histoire du climat depuis l'an mil, publié en 1967, est de ce point de vue pionnier. Ladurie a toutefois fait le choix d'écrire une « histoire climatique pure, affranchie de toute préoccupation ou présupposition anthropocentrique 2 », qui laissait volontairement de côté l'étude des rapports entre les fluctuations du climat et l'histoire humaine ${ }^{3}$. Ce faisant, il se plaçait en dehors du champ d'intervention de l'histoire environnementale, et même de l'histoire telle que définie par Marc Bloch par exemple, qui excluait de son champ d'étude les phénomènes naturels n'ayant eu aucun impact sur les sociétés humaines ${ }^{4}$. Sur la question des rapports entre climat et histoire humaine, Ladurie s'est longtemps montré très prudent, une attitude qu'il a révisée dans ses récentes publications ${ }^{5}$. Deux raisons principales peuvent expliquer le rôle mineur attribué au climat dans les affaires humaines : d'une part, à l'époque où Ladurie effectuait ses recherches dans les années 1960, la climatologie était encore une science balbutiante dont les résultats restaient très parcellaires. D'autre part, comme il le reconnaît ouvertement aujourd'hui, il redoutait de passer pour un naif déterministe aux yeux de ses confrères. Afin de ne pas endommager sa carrière, il a donc pratiqué l'autocensure et s'est vigoureusement démarqué de collègues attribuant une influence plus marquée au climat, comme le Suédois Gustav Utterström ${ }^{6}$.

Dans le prolongement des travaux de Ladurie, un deuxième type d'enquête de la climatologie historique consiste à s'interroger sur la vulnérabilité des sociétés et les réponses des groupes humains aux désordres climatiques (en particulier les épisodes catastrophiques ayant entraîné des crises de subsistance ou des périodes de bouleversements marqués). Cependant, pour des raisons sur lesquelles nous allons revenir, ces recherches relatives à l'impact du climat sur les sociétés humaines sont restées peu nombreuses et timides.

2. Le Roy Ladurie E., Histoire du climat depuis l'an mil, Paris, Flammarion, 1967, pp. 25-26.

3. Cette « histoire sans les hommes » visait principalement à récuser tout impact du climat sur l'histoire humaine, plutôt que le contraire, alors encore peu à l'ordre du jour. De ce fait, Ladurie choisit également systématiquement de ne pas tenir compte de sources indirectes (telles que les flux migratoires ou le prix des grains) pour reconstituer l'évolution du climat.

4. Bloch M., Apologie pour l'histoire ou métier d'historien, Paris, Armand Colin, 1949.

5. Le Roy Ladurie E., Histoire humaine et comparée du climat, Paris, Fayard, 2004-2009 (3 vol.).

6. «Interview d'Emmanuel Le Roy Ladurie » par F. Mauelshagen et al., Environment E History (à paraître en 2013). 
Une troisième source d'interrogation du passé touche à l'étude de la représentation du climat et du changement climatique. Elle invite à retracer les étapes de la découverte de l'effet de serre et ses mécanismes et à comprendre comment ce problème est devenu source d'inquiétude pour l'opinion publique en faisant appel à l'histoire des sciences, à l'histoire sociale, culturelle ou à l'étude des mouvements sociaux et de l'environnementalisme. Deux aspects différents, bien que connexes, ont été étudiés par les historiens : d'un côté, la question de la découverte du phénomène de réchauffement climatique global, c'est-à-dire une histoire du développement de la science qui a permis d'en mettre à jour les mécanismes invisibles. Il s'agit principalement d'écrire l'histoire d'un succès scientifique - donc une histoire optimiste - de la création de la discipline jusqu'à sa maturité contemporaine. De l'autre, l'intégration progressive du changement climatique parmi les problématiques de la cause environnementale et les tentatives visant à transformer le consensus scientifique en action politique. Là, la narration est plus pessimiste, puisque c'est essentiellement l'histoire d'un échec (tout au moins jusqu'à présent). On pourrait ajouter à cette catégorie quelques études récentes documentant par exemple les agissements des lobbies de l'énergie cherchant à semer la confusion dans l'esprit du public sur les dangers du réchauffement climatique depuis plus de 20 ans ${ }^{7}$.

Enfin, il existe de nombreuses autres études historiques ne portant pas spécifiquement sur le climat mais dont les problématiques sont cependant directement motivées par le réchauffement global actuel : c'est le cas par exemple de recherches visant à réévaluer le rôle des énergies fossiles dans l'histoire humaine récente - dans l'abolition de l'esclavage, par exemple - à l'heure où les problèmes climatiques nous obligent à envisager un avenir où ces énergies fossiles devront être utilisées avec plus de parcimonie. D’autres travaux réexaminent les essais passés de modification artificielle du climat et dont les effets souvent dangereux méritent d'être rappelés alors que la géo-ingénierie est souvent présentée comme une solution prometteuse ${ }^{8}$. D’autres encore réévaluent la politique de rationnement en Grande-Bretagne pendant la Seconde Guerre mondiale, pour voir quelles leçons peuvent en être tirées dans la perspective de l'établissement d'un rationnement ou d'une taxation du carbone ${ }^{9}$. Ajoutons qu'il existe une demande politique émergente pour ce type d'études.

Basé sur les études susmentionnées, cet article retrace tout d'abord la manière dont les hommes ont appréhendé le climat et ses variations au cours des siècles et montre que loin d'être un phénomène récent, la préoccupation des sociétés à cet égard est en réalité ancienne. Mais la prise de conscience

7. Oreskes N., Conway E. M., Les Marchands de doute, Paris, Le Pommier, 2012.

8. Fleming J. R., Fixing the Sky: The Checkered History of Weather and Climate Control, New York, Columbia University Press, 2010.

9. Roodhouse M., "Rationing returns: a solution to global warming?", History and Policy, mars 2007 (http://www.historyandpolicy.org/papers/policy-paper-54.html, consulté le 5 novembre 2012). 
contemporaine du réchauffement climatique anthropique traduit un changement d'échelle dans les inquiétudes, puisque pour la première fois se pose la question de la survie même de l'humanité (ou de la civilisation telle que nous la connaissons). Une seconde partie veillera à établir ce que l'histoire peut nous apprendre de la vulnérabilité des sociétés humaines face aux chocs climatiques. Enfin, l'article propose quelques réflexions sur les défis auxquels les historiens travaillant sur ces questions doivent faire face, à l'heure où le climato-scepticisme regagne du terrain, ainsi que sur les blocages qui entravent les recherches historiques en ce domaine.

\section{Hommes et climats : une histoire ancienne}

Examinons en premier lieu la manière dont les hommes ont conceptualisé le climat au cours des siècles derniers, jusqu'à la découverte relativement récente de l'effet de serre et du réchauffement climatique global. Quelques remarques préliminaires s'imposent. Tout d'abord, le sens du mot climat a luimême beaucoup évolué et a été l'objet de préoccupations de différentes catégories d'acteurs (philosophes, météorologistes, climatologues...). Jusqu'à ce que la climatologie moderne élabore au XIX ${ }^{\mathrm{e}}$ siècle les bases du concept de « climat » tel que nous l'entendons aujourd'hui, on avait « une idée purement thermique du climat qui était conçu comme une zone de latitude comprise entre deux parallèles raisonnablement proches, selon l'enseignement des astronomes grecs 10 ». Mais « au cours du XVII e siècle, le climat acquiert une certaine plasticité : s'il reste en partie déterminé par la position sur le globe, les discours savants - météorologiques et médicaux principalement - s’intéressent à ses variations locales, à ses innombrables altérations et au rôle de l'agir humain dans son «amélioration » ou sa «dégradation»11. Dans le présent texte, le mot « climat » s'entend dans son acception moderne d'ensemble des conditions météorologiques d'un lieu donné et il n'est pas fait de distinction forte entre des types de discours aux statuts parfois très différents car l'objectif est ici de dessiner les contours des discours, parfois très diffus, concernant le climat plutôt que de les analyser individuellement, en détail.

Il pourrait par ailleurs être utile d'étudier séparément la manière dont les hommes ont perçu leur relation avec le temps qu'il fait et leurs craintes concernant (a) la manière dont ils sont affectés par le climat et ses fluctuations et (b) la façon dont ils contribuent eux-mêmes à modifier le climat. Pourtant, dans les faits, ces trois aspects sont tellement imbriqués que tenter de les démêler gêne davantage la compréhension qu'elle ne la facilite, du moins jusqu'à la fin du XVIII' siècle. En effet, comme nous allons le voir, les inquiétudes concernant les changements climatiques sont intimement liées à la

10. Voir Pinna M., "Un aperçu historique de "la théorie des climats” ", Annales de géographie, vol. $98, n^{\circ} 547,1989$, pp. 322-325.

11. Locher F., Fressoz J.-B., «Le climat fragile de la modernité », La Vie des idées, 20 avril 2010 (http://www.laviedesidees.fr/Le-climat-fragile-de-la-modernite.html). 
manière dont les hommes ont considéré l'effet du régime climatique (le climat perçu comme figé et relativement immuable), autrement dit l'effet que les températures exercent sur les sociétés. Par ailleurs, dès qu'ils ont eu l'intuition qu'ils pouvaient modifier le climat - c'est-à-dire dès l'Antiquité - les hommes se sont inquiétés des conséquences que cela pouvait avoir sur eux.

Le changement climatique anthropique ne date sans doute pas de la révolution industrielle, même si celle-ci marque une nette accélération et un changement d'échelle. L'homme a pu - modestement - modifier les conditions climatiques localement et régionalement depuis fort longtemps, par le biais des travaux d'aménagement des sols (déforestation, drainage, etc.). Si l'on en croit les travaux du paléoclimatologue William Ruddiman, l'humanité aurait même eu une influence sur le climat global depuis la révolution néolithique. Par exemple, l'effondrement de la population amérindienne consécutive à l'arrivée des virus européens et à la brutalité des conquistadors après 1500 pourrait avoir joué un rôle dans le refroidissement climatique global subséquent connu sous le nom de petit âge glaciaire ${ }^{12}$. L'augmentation prodigieuse de la couverture forestière dans les Amériques (suite à l'abandon des cultures par plusieurs dizaines de millions d'Indiens), aurait en effet contribué à abaisser modestement la concentration de $\mathrm{CO}_{2}$ dans l'atmosphère ${ }^{13}$.

\section{Des craintes remontant à l'Antiquité}

L'homme a aussi conscience depuis longtemps de l'impact de ses actes à l'échelle régionale. L'une des premières constatations empiriques a été celle des relations entre couverture forestière et précipitations. Ainsi, le ou les auteurs de l'épopée de Gilgamesh - l'un des plus anciens textes conservés, inspiré de récits composés en Sumérien vers le III millénaire avant J.-C. - redoutaient déjà que les déforestations en Mésopotamie entraînent une baisse des précipitations 14. Théophraste, un philosophe contemporain et disciple d'Aristote fut quant à lui le premier à s'interroger sur la possibilité pour l'homme de modifier artificiellement les températures d'un milieu local 15. Théophraste n'évoquait que des microclimats locaux, mais il ne fait pas de doute que pour le disciple d'Aristote, ils révèlent des grands principes à l'œuvre, qui montrent que l'homme peut modifier le climat. Cette idée, dont on retrouve les traces dans les écrits d'Albert Le Grand au XIII' siècle ou encore

12. Généralement, la période du petit âge glaciaire s’étend de 1550 à 1850 , mais certains auteurs font remonter le début du refroidissement à 1300 .

13. Ruddiman W. F., Plows, Plagues, and Petroleum: How Humans Took Control of Climate, Princeton, N.J., Princeton University Press, 2005. Ses travaux ne font pas l'objet d'un consensus scientifique et n'excluent pas d'autres facteurs naturels. Pour le petit âge glaciaire, une diminution de l'activité solaire et des éruptions volcaniques ont joué un rôle sans doute plus important que la reforestation américaine.

14. Grove R. H., Green Imperialism: Colonial Expansion, Tropical Island Edens and the Origins of Environmentalism, 1600-1860, Cambridge, Cambridge University Press, 1995, p. 18.

15. Hughes J. D., “Theophrastus as Ecologist”, Environmental Review, vol. 9, n4, 1985, p. 302. 
de Christophe Colomb, traversa les siècles et fit l'objet de très nombreuses discussions en particulier aux XVIII et XIXe siècles, nous y reviendrons.

Historiquement, ce ne sont pourtant pas les changements climatiques (naturels ou anthropiques) qui ont d'abord et durablement intrigué ou inquiété les hommes. Jusqu'à une période récente, c'est surtout le rôle joué par les régimes climatiques sur les caractères, les mœurs ou le développement des hommes et des nations qui faisait l'objet de débats passionnés. Il faut dire quelques mots sur cette " théorie des climats », car elle a récemment resurgi dans les débats sur les conséquences du changement climatique.

La croyance selon laquelle le climat détermine les possibilités humaines futures et explique les différences entre les hommes et les sociétés n'est pas nouvelle. On peut retracer cette idée au moins jusque dans l'œuvre attribuée à Hippocrate, Des Airs, des eaux et des lieux (environ 400 av. J.-C.) - même si ni Hippocrate ni ses successeurs n'utilisent le mot «climat » dans son sens moderne, météorologique. Dans ce traité, l'auteur met toutefois en évidence l'action du climat sur les qualités morales et intellectuelles des différents groupes humains. Aristote reprend à son compte cette théorie dans $\mathrm{La}$ Politique. Inaugurant une tradition qui fera beaucoup d'émules, Aristote considère que le meilleur climat est celui où il habite 16 ! Cette " théorie des climats » a connu ensuite un fort retentissement. Il est bien sûr impossible d'en retracer ici tous les avatars, mais on comprend dès lors qu'à partir du moment où les hommes ont réalisé qu'ils pouvaient intervenir sur leur climat, ils se sont interrogés sur les effets positifs ou négatifs que cela pouvait entrâ̂ner non seulement pour l'environnement, mais aussi pour eux-mêmes ${ }^{17}$. Les considérations sur les conséquences climatiques des coupes de bois sont fréquentes à l'époque moderne. En France, ces idées furent remises au goût du jour par Jean Bodin au XVIe siècle, puis cristallisées au XVIII siècle par l'abbé Jean-Baptiste Dubos dans ses Réflexions critiques sur la poésie et sur la peinture (publiées en 1719), et surtout, bien sûr, par Montesquieu dans L'Esprit des Lois. Dans le passage qui lui fut le plus reproché par ses détracteurs, le baron de La Brède écrivait :

«Il ne faut donc pas être étonné que la lâcheté des peuples des climats chauds les ait presque toujours rendus esclaves, et que le courage des peuples des climats froids les ait maintenus libres. C'est un effet qui dérive de sa cause naturelle. 18 »

16. Aristote, Politique, livre IV (VII), chapitre VI, traduction de J. Barthélemy-Saint Hilaire, Paris, Librairie philosophique de Ladrange, 1874 (http://remacle.org/bloodwolf/philosophes/Aristote/politique4.htm\#VI, consulté le 12 novembre 2012).

17. Glacken C. J., Traces on the Rhodian shore, op. cit.

18. Montesquieu, L'esprit des lois, Genève, Barrillot \& Fils, 1748 (3e partie, Livre XVIII, chap. II). 
Montesquieu s'inquiétait aussi des risques encourus par les hommes quand ils changeaient de régime climatique, le mieux étant selon lui de rester dans sa région de naissance. Toutefois, il reconnaissait que l'ingéniosité humaine, l'éducation, le gouvernement, la médecine ou l'agriculture pouvaient supplanter les influences négatives du climat. Les thèses de Montesquieu furent reprises en particulier par Buffon dans son Histoire naturelle publiée en 1749, puis par David Hume ${ }^{19}$. Ces théories étaient répandues dans toutes les couches de la population et influençaient notamment les choix d'émigration 20 .

À la fin du XVIII" siècle, selon l'historien James Fleming, un grand nombre parmi les élites des Lumières partageaient les idées suivantes : les différences culturelles entre les hommes sont déterminées ou fortement influencées par le climat ; l'Europe est devenue plus tempérée depuis l'Antiquité grâce aux défrichements et à l'expansion de l'agriculture, et le climat américain va suivre la même trajectoire heureuse car favorable à l'épanouissement des civilisations avancées ${ }^{21}$.

À partir de la fin du XVIII e siècle, il convient de faire une distinction entre l'histoire de cette « théorie des climats » (qui concerne l'influence du climat sur les hommes), et l'histoire de la (re)découverte de l'influence des hommes sur le climat, car ces deux paradigmes vont connaître des évolutions assez distinctes, avant de se télescoper à nouveau à la fin du $\mathrm{XX}^{\mathrm{e}}$ siècle.

\section{Le lent reflux des peurs}

Au début du XIX ${ }^{\mathrm{e}}$ siècle, les débats concernant les changements de climat deviennent plus scientifiques. Pour autant, les théories préscientifiques ne disparaissent pas du jour au lendemain. On continue à penser à la fin du XVIII siècle, en suivant Buffon, que la déforestation est bénéfique pour le climat en France. Après la Révolution, un changement de perception s'opère : couper des arbres n'est plus considéré comme bénéfique du point de vue du climat. On se met au contraire à craindre des accidents météorologiques dont les causes sont imputées aux paysans en raison des dommages causés par ceux-ci à la forêt pendant la Révolution ${ }^{22}$. En 1821, le ministre de l'Intérieur envoie ainsi une circulaire aux préfets attribuant un présumé refroidissement aux défrichements des forêts, sans doute un prétexte commode pour reprendre en

19. En particulier dans son essai “Of the Populousness of Ancient Nations”, publié pour la première fois vers 1750. Cf. Hume D., Essays: Moral, Political and Literary, ed. T. H. Green and T.H. Grose, London, 1875, 1, pp. 432-433.

20. Mouhot J.-F., Les Réfugiés acadiens en France (1758-1785) : l'impossible réintégration ?, Rennes, Presses universitaires de Rennes, 2012 (première édition, Québec, Septentrion, 2009).

21. Fleming J. R., Historical Perspectives on Climate Change, New York, Oxford University Press, 1998, p. 18.

22. Pendant la Révolution, la forêt, auparavant très protégée par l’État, a souffert de la désorganisation de l'administration (suppression des maîtrises des Eaux et Forêts...). Les paysans furent blâmés pour les déprédations. 
main le contrôle de celles-ci. L'idée reste dans l'air du temps : en 1836, François Arago s'oppose à la levée des restrictions sur l'exploitation du bois par crainte des effets sur le climat, même s'il reconnaît qu'il n'existe aucune certitude à ce sujet. Plus avant dans le XIXe siècle, des théoriciens de la colonisation affirment que l'aridité de certains pays comme l'Égypte est due à la mauvaise gestion de l'environnement par les autochtones, ce qui a conduit à une détérioration du climat - susceptible d'être à son tour corrigée par un « reboisement $» 23$.

$\mathrm{Au}$ cours du XIXe siècle, on observe un reflux progressif de la croyance dans l'influence humaine sur le climat. Celle-ci avait d'ailleurs toujours été sujette à controverse devant l'absence de preuves scientifiques. Ce repli s'opère principalement suite à la collecte simultanée, dans de nombreux pays occidentaux, d'une grande quantité de données météorologiques rigoureusement tabulées, cartographiées et analysées avec la volonté de repérer des régularités. La multiplication de ces données conjuguée à l'intensification et à la globalisation des échanges, favorise l'établissement de la climatologie scientifique dans le courant du XIXe siècle; le discours "impressionniste » des Lumières s'efface au profit de séries statistiques solides. Plusieurs voix s'élèvent à ce moment pour remettre en cause l'idée d'une influence générale des hommes sur le climat. Dès 1799, dans un essai publié aux États-Unis, Noah Webster évoque les problèmes méthodologiques et l'absence de preuves avancées par les promoteurs d'une influence anthropique sur le climat. En 1842, Samuel Forry publie The Climate of the United States and its Endemic Influences et conclut, sur des bases désormais solides, que ceux qui ont parlé d'un réchauffement du pays se sont trompés ${ }^{24}$. Les conclusions de Forry, louées en particulier par le naturaliste et explorateur allemand Alexandre von Humboldt, gagnent du terrain tout au long du XIXe siècle. L'idée que l'homme transforme le climat recule, notamment en raison de nouvelles recherches qui établissent l'origine non humaine des cycles glaciaires. Ironie de l'histoire : alors que, sous l'effet de la révolution industrielle et de l'augmentation des émissions de $\mathrm{CO}_{2}$, le réchauffement global s'enclenchait, les savants s'accordaient de plus en plus à dire que les fluctuations climatiques relevaient de facteurs naturels et non anthropiques. Ce paradigme allait dominer les sciences pendant plus d'une centaine d'années.

\section{L'apport de la science climatique}

Pourtant, c'est dans ce contexte qu'un savant français, Joseph Fourier, publie en 1824 sa théorie de l'effet de serre pour expliquer la température curieusement élevée de l'atmosphère terrestre. Initialement peu commentés, ses travaux sont repris et complétés par plusieurs scientifiques dans la seconde

23. Locher F., Fressoz J.-B., "Le climat fragile de la modernité », art. cit.

24. Fleming J., Historical Perspectives, op. cit., p. 49. 
moitié du XIX e siècle. Le Britannique John Tyndall démontre que des gaz invisibles peuvent absorber et émettre de la chaleur dans une série d'articles publiés à partir de 1859 25. En 1896, le Suédois Svante Arrhenius émet quant à lui l'hypothèse qu'une augmentation de la concentration atmosphérique en $\mathrm{CO}_{2}$ entraînerait des rétroactions pouvant expliquer l'avancée et le recul des âges glaciaires ${ }^{26}$. Au cours du XIX ${ }^{\mathrm{e}}$ siècle, ces recherches restaient marginales et ne suscitaient pas d'inquiétude, même chez les scientifiques qui étudiaient ces phénomènes. En 1908, dans un livre où il estimait qu'un doublement de la concentration de $\mathrm{CO}_{2}$ pourrait provoquer un réchauffement climatique global de $4^{\circ} \mathrm{C}$, il jugeait que cela serait bénéfique pour l'humanité 27 .

Nous ne pourrons évoquer ici que de manière extrêmement succincte l'histoire de la science climatique et de la problématisation du réchauffement global. Les étapes de la découverte et de la politisation du réchauffement anthropique contemporain ont été bien jalonnées par plusieurs études récentes ${ }^{28}$. Dès 1938, un climatologue amateur britannique, G.S. Callendar argumentait devant la Royal Meteorological Society qu'un réchauffement était en cours et que les hommes en étaient responsables par leurs émissions de gaz à effet de serre ${ }^{29}$. Mais au cours de la première moitié $\mathrm{du} \mathrm{XX}^{\mathrm{e}}$ siècle, la plupart des scientifiques ne pensaient pas qu'une augmentation de la concentration de $\mathrm{CO}_{2}$ entraînerait un réchauffement climatique planétaire. Ce n'est qu'après la Seconde Guerre mondiale que le changement climatique fait irruption dans les débats publics. La guerre froide incite en effet les États-Unis à investir massivement dans la recherche atmosphérique, au moment où ont lieu les premières tentatives de modifications artificielles du climat. Les chercheurs redécouvrent les travaux de Callendar ou Arrhenius. C'est dans ce contexte qu'en 1956 et 1957, trois scientifiques nord-américains (Gilbert Plass, Roger Revelle et Hans Suess), inquiets de l'accumulation de $\mathrm{CO}_{2}$ dans l'atmosphère, tirèrent pour la première fois la sonnette d'alarme en signalant que l'homme était en train de conduire une expérience inédite à grande échelle sur l'atmosphère. Mais l'heure n'était pas encore à l'alarmisme. Par ailleurs, le mouvement environnemental moderne, qui allait prendre le relais pour informer le grand public sur le réchauffement climatique anthropique, n’en était alors qu’à ses

25. Voir la liste des articles publiés par Tyndall dans Fleming J., Historical Perspectives, op. cit., pp. 178-79

26. Arrhenius S., "On the Influence of Carbonic Acid in the Air Upon the Temperature of the Ground" The London, Edinburgh and Dublin Philosophical Magazine and Journal of Science 41, no. 251 (1896), pp. 237-276.

27. Il semble toutefois qu'Arrhenius ait été le premier à évoquer la possibilité (si ce n'est le terme) de réfugiés climatiques (Hulme M., "Reducing the Future to Climate”, Osiris, vol. 26, n¹, 2011, pp. 245-266).

28. Voir en particulier Weart S. R., The Discovery of Global Warming, Cambridge, Harvard University Press, 2003 ; Howe J. P., Making Global Warming Green, thèse de doctorat en histoire, Stanford, 2010.

29. Callendar, G.S. "The Artificial Production of Carbon Dioxide and Its Influence on Temperature”, Quarterly Journal of the Royal Meteorological Society, 64, no. 275 (1938), pp. 223-240. 
balbutiements ${ }^{30}$. Le mouvement écologiste ne commencerait à acquérir une certaine visibilité que dans les années 1960 et il faudrait encore attendre avant que ce mouvement émergent ne se saisisse du problème climatique. Pendant un court moment, cependant, ce sont des craintes liées à un possible refroidissement du climat qui font brièvement la une des journaux ${ }^{31}$.

Les années 1980 constituent une décennie charnière alors que l'effet de serre fait pour la première fois la une du New York Times en 1981 32. Il faut dire qu'entre-temps, le trou de la couche d'ozone, très médiatisé à l'époque, montre que l'homme peut avoir un impact sur l'atmosphère plus important qu'il ne l'imaginait. Mais le greenhouse effect ne devient véritablement un sujet d'inquiétude pour l'opinion publique américaine qu'en 1988. En juin de cette année-là, James Hansen, un climatologue de la NASA affirme devant le congrès américain qu'il est certain à $99 \%$ que le climat se réchauffe et que les hommes en sont responsables. Son témoignage fait grand bruit. Des déclarations tonitruantes de Margaret Thatcher et Ronald Reagan conduisent alors à un emballement médiatique sans précédent et à une vive prise de conscience de l'opinion partout dans le monde occidental et au-delà. Ainsi, en 1989, un sondage montre que $79 \%$ des Américains ont entendu parler de l'effet de serre, contre $38 \%$ en $1981{ }^{33}$. Les écologistes font des percées remarquables dans la plupart des pays d'Europe. En réponse aux questions et aux inquiétudes, le groupe d'experts intergouvernemental sur l'évolution du climat (GIEC) est fondé par l'ONU en 1988.

Pourtant, au début des années 1990, la couverture médiatique de ces questions s'estompe considérablement, malgré le Sommet de la terre à Rio en 1992 établissant la Convention-cadre des Nations Unies sur les changements climatiques. La première guerre du Golfe, la chute du mur de Berlin, la crise économique détournent l'attention; les journalistes et les lecteurs se lassent d'un sujet qui tarde à se manifester concrètement ${ }^{34}$. Mais ce recul est aussi le résultat d'un effort délibéré des lobbies de l'énergie pour semer le doute dans les esprits en exagérant les incertitudes scientifiques. Les négociations internationales progressent lentement au cours de la période. En 1997, le protocole de Kyoto est signé mais il ne sera pas ratifié par le principal pollueur, les ÉtatsUnis, et n'entrera en vigueur en 2005 qu'après une adhésion tardive de la Russie.

30. Guha R., Environmentalism: A Global History, New York, Longman, 2000.

31. Le refroidissement des températures globales entre 1950 et 1970 est dû au phénomène du global dimming (assombrissement global ou obscurcissement planétaire).

32. Sullivan W., "Study Finds Warming Trend That Could Raise Sea Levels", New York Times, August 22, 1981.

33. Cité par Weart S., The Discovery of Global Warming, Cambridge, Harvard University Press, 2003.

34. Sur cet épisode d'emballement médiatique de courte durée, voir Mouhot J.-F., McKay J., Hilton M., «Le Greenrush. Essai d'interprétation de la "bulle verte" dans les années 1980 ", Vingtième Siècle, n¹13, janvier-mars 2012, pp. 67-81. 
On peut déceler un nouveau sursaut dans le processus de définition du problème à partir du début du millénaire en Europe et de 2005 aux ÉtatsUnis : les inondations britanniques de 2000, la canicule de 2003 en Europe, l'ouragan Katrina en 2005, attribués de manière croissante au réchauffement climatique global, tout comme le rapport Stern en 2006, ramènent nécessairement le problème sur le devant de la scène. Le succès du film Une Vérité qui dérange d'Al Gore (prix Nobel de la Paix avec le GIEC en 2007), les rapports de plus en plus alarmistes des climatologues et la mobilisation croissante des ONG contribuent à ramener fermement la question au premier plan médiatique. Par exemple, l'organisation du Grenelle de l'environnement en France en 2007 ou le vote du Climate Change Act en 2008 en Grande-Bretagne témoignent de l'inquiétude du grand public pour ces questions. En Australie et aux États-Unis, des gouvernements favorables à une action envers le changement climatique succèdent à des gouvernements qui y étaient hostiles.

\section{Rebondissements récents}

Mais depuis 2008, on assiste à nouveau à un recul des préoccupations relatives au changement climatique, alors même que la concentration de $\mathrm{CO}_{2}$ dans l'atmosphère continue de croître à un rythme soutenu. Plusieurs raisons, souvent similaires à celles ayant caractérisé la période post 1988-1989, peuvent expliquer ce désintérêt. La crise économique et financière depuis 2007 a joué un rôle de premier plan, tout comme l'échec de la conférence de Copenhague fin 2009 35. En 2009, l'affaire connue sous le nom de "Climategate» a également contribué à renforcer dans une partie de l'opinion le sentiment que la science climatique était peu fiable ${ }^{36}$. L'accident nucléaire de Fukushima en 2011 a redirigé l'attention sur le nucléaire. Mais d'autres raisons plus profondes sont également à l'œuvre pour expliquer ce désintérêt, qui se traduit par une baisse très nette du nombre d'Occidentaux estimant les hommes responsables du réchauffement climatique global. La France n'est pas épargnée par ce grand retour du climato-scepticisme ${ }^{37}$. La figure la plus médiatique en est certainement l'ancien ministre de l'Éducation nationale Claude Allègre, mais de nombreux autres sceptiques ou flirtant avec cette tendance en ont profité pour faire entendre leur voix, et parmi eux, quelques essayistes et chercheurs en sciences humaines - comme Pascal Brückner ou Pascal Acot ${ }^{38}$.

35. Pour une interprétation alternative du sommet de Copenhague, voir Jancovici J.-M., Changer le Monde! Tout un programme, Paris, Calmann-Levy, 2011.

36. En Novembre 2009, plusieurs milliers d'e-mails provenant de chercheurs du Climatic Research Unit de l'Université de East Anglia furent divulgués sur internet, certains mettant apparemment en cause l'intégrité de certains climatologues. Plusieurs enquêtes ont par la suite blanchi les scientifiques concernés. Sur cette "affaire", voir Pearce F., The Climate Files, Londres, Guardian Books, 2010.

37. Godard O., "Le Climato-scepticisme médiatique en France : un sophisme moderne ", Cabiers n²011-20, département d'économie de l'École Polytechnique.

38. Allègre C., L'Imposture climatique, Paris, Plon, 2010 ; Bruckner P., Le fanatisme de l'apocalypse : sauver la terre, punir l'homme, Paris: Grasset, 2011 ; Acot P., Climat, un débat dévoyé ?, Paris, Armand Colin, 2010. 
$\mathrm{Au}$ terme de cette présentation très rapide, un premier bilan s'impose. Comme nous l'avons vu, les hommes réfléchissent depuis fort longtemps à leurs rapports au climat et s'inquiètent depuis presque autant de temps des changements climatiques et de leurs conséquences potentielles. Dipesh Chakrabarty, dans un texte abondamment commenté, estime que les historiens de l'environnement ont longtemps été incapables de considérer les hommes autrement que comme «prisonniers du climat »- une expression empruntée à Braudel -, plutôt que comme des agents capables de modifier le climat. ${ }^{39}$ Or si cette opinion était celle d'une majorité des scientifiques au début du siècle dernier, il en fut autrement pour la plupart des hommes, la plupart du temps. Certes, l'humanité se préoccupe depuis longtemps des changements climatiques, mais il y a aujourd'hui plusieurs nouveautés. Tout d'abord, c'est la première fois qu'un consensus scientifique notoire est établi à la fois sur les causes et les effets du réchauffement climatique anthropique (dont le caractère potentiellement catastrophique est régulièrement souligné par les chercheurs). Les cris d'alarme des scientifiques dénotent un changement de paradigme puisque pour la première fois il est question du futur même de l'humanité. Ensuite, l'amplitude du réchauffement annoncé est sans précédent dans l'histoire humaine récente. Dans cette configuration, une démarche historique ne peut que partiellement nous aider à anticiper les problèmes générés par le réchauffement climatique actuel.

\section{Ce que l'histoire peut nous apprendre}

Dans un article publié en 2008, John McNeill se demandait si l'histoire pouvait nous aider face au défi climatique. Sa réponse était mitigée : dans un monde qui pourrait se réchauffer de six degrés au cours de ce siècle, McNeill commence par rappeler que « s'il y a eu, au cours de l'histoire de la planète, des épisodes analogues au changement climatique en cours, il n'en existe pas dans l'histoire humaine. Nous sommes en territoire inconnu » 40. En effet, si le climat de la terre n'a pas cessé de fluctuer au cours des 2,7 millions d'années passées, alternant entre des cycles glaciaires et interglaciaires, il n'y a pas eu de variations majeures du climat depuis la dernière glaciation, qui s'est terminée il y a 12000 ans. En conséquence, l'histoire ne peut pas nous apprendre grandchose sur les réponses politiques et sociales à apporter à un réchauffement climatique sans précédent dans l'histoire humaine.

39. Chakrabarty D., "The climate of history: Four theses”, Critical Inquiry, vol. 35, n², 2009, pp. 197-222.

40. McNeill, J., "Can History Help Us with Global Warming?”, in Campbell K. (ed), Climatic Cataclysm: The Foreign Policy and National Security Implications of Climate Change, Washington, Brookings Institution Press, 2008, pp. 26-48. 
Même s'il est toujours difficile, et a fortiori dans ces circonstances, de s'appuyer sur la connaissance du passé pour anticiper ce qui pourrait advenir dans un monde en réchauffement rapide, il est néanmoins utile de pouvoir observer les effets socio-politiques (autant qu'on puisse les connaître) des changements climatiques passés sur les sociétés. Comme nous l'avons déjà noté, les études sur ce sujet sont relativement rares, en raison notamment de l'inhibition opérée par la peur du déterminisme, mais aussi parce que les changements climatiques passés étaient la plupart du temps d'une amplitude suffisamment faible pour ne pas être détectés (les températures de l'hémisphère nord, notamment, ne fléchirent que de moins de $1^{\circ} \mathrm{C}$ en moyenne pendant le petit âge glaciaire). Pour ces raisons, les conséquences sur les sociétés ne sont pas toujours faciles à identifier, et la plupart des effets attribués au climat sont vigoureusement discutés par les spécialistes. Sans prétendre évidemment à une quelconque exhaustivité, nous pouvons cependant évoquer quelques périodes-clés, en commençant par la préhistoire. La fin du dernier âge glaciaire coïncide en effet avec le début de l'agriculture et l'émergence de sociétés plus complexes. Mais les archéologues sont divisés entre deux écoles, l'une pour qui l'agriculture résulte de l'amélioration générale du climat correspondant au début de l'Holocène (il y a 12000 ans), l'autre pour laquelle l'agriculture est au contraire une innovation que nos ancêtres furent forcés d'adopter face à la dégradation du climat pendant la (relativement brève) période du Dryas récent (12 700 et $11500 \mathrm{BP})^{41}$. Ce débat met implicitement en jeu une question plus fondamentale, naïve au premier abord : les changements climatiques sont-ils fondamentalement mauvais ou peuvent-ils au contraire impulser des innovations "utiles » au genre humain 42 ? L'archéologue Brian Fagan attribue par exemple un rôle de stimulant au petit âge glaciaire dans le déclenchement de la révolution agricole en Grande-Bretagne à partir du milieu du XVIII ${ }^{\mathrm{e}}$ siècle ${ }^{43}$.

L'organisation des sociétés selon des systèmes politiques et économiques de plus en plus complexes a eu un effet paradoxal quant à leur résistance aux chocs climatiques. En effet, comme l'ont montré de nombreux archéologues, les sociétés complexes sont moins vulnérables aux faibles variations annuelles

41. BP : Before present (time), c'est-à-dire avant le temps présent, correspondant en archéologie préhistorique par convention à l'année 1950. L'importance du réchauffement climatique au cours de l'Holocène se mesure peut-être principalement par le fait qu'elle a permis une extension de l'agriculture en dehors de la zone intertropicale (Christian D., Maps of Time: An Introduction to Big History, Berkeley, University of California Press, 2005).

42. Je m’appuie ici principalement sur l'excellent article synthétique de White S., "Climate Change in Global Environmental History”, in McNeill J. R., Mauldin E. S. (eds), A Companion to Global Environmental History, Chichester, Blackwell, 2012, pp. 395-403. Sauf indication contraire, les références des différents exemples et arguments avancés dans les paragraphes qui suivent proviennent toutes de cet article.

43. Fagan B. M., The Little Ice Age: How Climate Made History, 1300-1850, New York, Basic Books, 2000. 
de climat, mais encourent de graves catastrophes lors de perturbations climatiques plus importantes, de même que la construction de digues le long d'une rivière permet d'éviter de petites inondations mais accroît le risque de problèmes sérieux si ces digues venaient à céder. De nombreux archéologues estiment donc fort plausible qu'un dérèglement du climat autour de 2200 avant J.-C. soit à l'origine de crises économiques et politiques en Égypte, Syrie, Mésopotamie, et peut-être aussi en Chine. Plus tard, la montée et l'apogée de l'Empire romain coïncident avec une période d'optimum climatique autour de la Méditerranée qui s'achève vers la fin du III e siècle. La détérioration subséquente pourrait avoir joué un rôle dans la chute de l'Empire romain d'Occident, tout comme dans le déclin de la population et de l'agriculture européennes au cours du Haut Moyen Âge. Quelques siècles plus tard, les Mayas pourraient aussi avoir été victimes d'un changement climatique, suite à une série d'éruptions volcaniques qui ont probablement contribué à des sécheresses sévères dans le Yucatan. Même si la question, popularisée par Jared Diamond, fait l'objet de débats animés parmi les spécialistes, des indices assez nombreux indiquent que ces sécheresses - combinées à d'autres problèmes environnementaux - ont fragilisé la production agricole des Mayas et entraîné une forte dépopulation et l'abandon de la plupart des villes ${ }^{44}$. De la même manière, aux XIVe et XVe siècles, des sécheresses sévères pourraient avoir contribué à la chute d'Angkor, la capitale du royaume Khmer.

En Europe, pendant une période relativement chaude - comparée à ce qui précédait et ce qui suivit - connue sous le nom d'optimum climatique médiéval, entre 1000 et 1300 environ, la population augmenta rapidement et bénéficia largement des meilleures conditions climatiques (et du développement progressif de dizaines de milliers de moulins à eau), qui favorisèrent probablement le développement des villes. Mais un refroidissement du climat vers 1300 (considéré par certains comme le début du petit âge glaciaire, la dernière anomalie climatique globale avant l'époque contemporaine) et une succession d'étés humides eurent un effet désastreux sur les récoltes et furent directement responsable de la grande famine de 1315-1317. Ce dérèglement du temps contribua probablement à fragiliser les populations et à les rendre plus vulnérables à l'épidémie de peste noire (à partir de 1348 en France) qui tua au moins un tiers de la population du continent et de la Chine, où la dynastie Yuan aurait aussi été une victime collatérale du changement climatique. Les Vikings installés au Groenland pendant la période de relatif réchauffement après l'an mil firent aussi les frais du refroidissement ultérieur. Leur cas a été à nouveau étudié de manière particulièrement détaillée par Jared Diamond ${ }^{45}$, qui a érigé

44. Dans son livre Effondrement. Comment les sociétés décident de leur disparition ou de leur survie (Paris, Gallimard, 2006), Jared Diamond estime que le climat est au moins en partie responsable de l'effondrement de plusieurs sociétés, comme les Vikings du Groenland, les Mayas ou les Anasazis du Sud-Ouest des actuels États-Unis. Pour une critique, voir McAnany P. A., Yoffee N. (eds), Questioning Collapse. Human Resilience, Ecological Vulnerability, and the Aftermath of Empire, Cambridge, Cambridge University Press, 2010.

45. Diamond J., Effondrement..., op. cit. 
ces groupes Vikings en parabole destinée à souligner le danger du conservatisme et l'importance de la flexibilité et de l'innovation face au changement. Toutefois, d'autres auteurs ont souligné que les sociétés vikings du Groenland avaient été résilientes plus longtemps que certaines sociétés contemporaines érigées en exemple par Diamond, comme la République dominicaine.

Le petit âge glaciaire a été de ce fait le plus étudié de tous les phénomènes climatiques passés. Loin de constituer un épisode uniformément froid, il fut ponctué de nombreuses variations parfois très rapides. Le phénomène est particulièrement bien documenté dans le nord de l'Europe. Les effets se firent également sentir de manière particulièrement marquée en Chine et dans l'Empire Ottoman, où les disettes contribuèrent à des troubles politiques majeurs.

En Europe, les épisodes combinant dérèglement climatique, mauvaises récoltes, famines et augmentation significative de la mortalité sont particulièrement bien documentés pour la fin du XVIe siècle et durant la crise générale du XVII ${ }^{e}$ siècle. Le Roy Ladurie estime ainsi que le petit âge glaciaire a pu concourir à l'effroyable mortalité de la guerre de Trente Ans (1618-1648) et à l'éclosion de troubles politiques ayant contribué à la Fronde ou à la première guerre civile anglaise (1642-1646) ${ }^{46}$. Certains chercheurs se sont également penchés sur les mutations culturelles engendrées par le petit âge glaciaire, sur les vêtements ou l'architecture. L'un des exemples récents les plus souvent cités (et les plus critiqués) est celui de l'influence du refroidissement climatique sur les procès en sorcellerie, les « sorcières » étant accusées de dérégler le climat 47 .

Plus récemment encore, certains historiens ont attribué un rôle décisif à des épisodes climatiques particulièrement sévères, comme celui qui contribua aux mauvaises récoltes en France à la fin des années 1780, précipitant ainsi la crise financière et les troubles à l'origine de la réunion des États généraux et de la Révolution en France. Le Roy Ladurie souligne le rôle historique des crises de subsistance, "des moments de baisse de la production agricole, participant souvent à la concrétisation de troubles sociaux en latence jusqu'alors ». S’il estime que le climat ne figure pas parmi les déterminants essentiels de la Révolution, "dans le court terme, les facteurs non point de causalité proprement dite mais de provocation, comme ceux que déclenche un commutateur ou un pistolero, ont certainement joué 48 ». Le climat pourrait aussi avoir joué un rôle indirect non négligeable (via le déclenchement d'autres crises de sub-

46. Le Roy Ladurie E., Histoire Humaine et comparée du climat, vol. 1, Paris, Fayard, 2004, chapitre 8.

47. Behringer W., A Cultural History of Climate, Cambridge, Polity Press, 2007. Pour une critique, voir Mauelshagen F., Histoire du climat, 1500-1900, Paris, Hermann (à paraître en 2013).

48. Le Roy Ladurie E., « Révolutions, le déclic climatique », Sciences Humaines, Grand Dossier $\mathrm{n}^{\circ} 25,2011-2012$, pp. 8-11. 
sistance) dans les révolutions de 1830 et de 1848, ainsi que dans les famines de la fin du XIX siècle en Inde, en Chine et au Brésil qui ont fait plusieurs dizaines de millions de victimes ${ }^{49}$.

Que conclure de ce bref tour d'horizon ? Il y a de bonnes raisons de penser, comme le suggère Sam White, que « le climat a été une force puissante bien que souvent négligée - dans l'histoire humaine. De la préhistoire jusqu'à l'époque contemporaine, les changements climatiques ont joué un rôle dans l'évolution humaine et les migrations, l'essor et la chute de civilisations, le succès ou l'échec de colonies et la stabilité ou la crise d'États et d'Empires 50 ». Au terme de la présentation d'un vaste panorama de l'histoire humaine prêtant une attention particulière aux phénomènes climatiques, John Brooke va encore plus loin et argumente contre Malthus que l'effondrement de sociétés dans les siècles passés s'explique non par des facteurs endogènes (le fameux piège ou dilemme malthusien), mais presque toujours par des facteurs exogènes (climatiques en particulier) ${ }^{51}$.

Cependant, il est manifestement malaisé d'attribuer précisément au climat telle crise ou difficulté du passé. Le rôle des fluctuations climatiques a souvent été complexe et indirect et dépend de nombreux autres facteurs difficiles à isoler de façon précise. Les liens de causalité ne sont pas évidents à établir, ce qui rend laborieuse toute conclusion définitive sur le rôle exact du climat dans l'histoire humaine (ce n'est pas par hasard si, dans son récent tour d'horizon de la question, Sam White est constamment contraint d'utiliser le conditionnel lorsqu'il attribue un rôle au climat ${ }^{52}$ ). Les dérèglements climatiques font plus souvent fonction de déclencheur. Mais rappelons encore une fois que le réchauffement climatique actuel pourrait entraîner des variations de températures quatre ou cinq fois plus élevées que celles qu'ont connues nos ancêtres au cours de l'histoire humaine.

\section{L'histoire peut-elle nous aider à y voir plus clair?}

L'histoire ne peut-elle donc rien nous apprendre d'utile pour affronter les crises climatiques à venir ? Si l'on élargit l'angle d'approche en étudiant de façon plus générale les catastrophes «naturelles », la connaissance du passé peut offrir un autre éclairage intéressant ${ }^{53}$. En effet, toutes les sociétés ont été, à un moment ou un autre, affectées par des désastres ; comprendre les réponses qui y ont été apportées peut fournir un indicateur précieux de celles

49. Davis M., Génocides tropicaux. Catastrophes naturelles et famines coloniales, Paris, La Découverte, 2003.

50. White S., "Climate Change in Global Environmental History”, art. cit.

51. Brooke J. L., A Rough Journey: Human History and a Volatile Earth, New York, Cambridge University Press (à paraître).

52. White S., "Climate Change in Global Environmental History”, art. cit.

53. Les études récentes dans le champ des disaster studies montrent qu'aucune catastrophe n'est entièrement naturelle, et comprend toujours un important élément humain. 
auxquelles on peut s'attendre dans le futur. Enfin, l'histoire peut aussi nous fournir d'importants enseignements sur la manière dont se déroulent les transitions technologiques et énergétiques (l'une ou l'autre étant nécessaire pour sortir de l'impasse où nous nous trouvons actuellement). Le passé peut donc suggérer des pistes pour accroître la résistance et la résilience de nos sociétés face aux désordres climatiques annoncés ${ }^{54}$.

Au cours de l'histoire humaine, les principales catastrophes naturelles ayant affecté les hommes ont sans conteste été, d'un point de vue strictement numérique, les épidémies. Certaines ont pu causer, dans les cas les plus sévères, entre 30 et 100 millions de morts (peste noire de 1348-52, épidémies post-colombiennes en Amérique, grippe espagnole de 1918-1919). Par comparaison, les pires sécheresses comme les pires inondations n'ont tué jusqu'à aujourd'hui «que » quelques millions d'humains (entre deux et cinq millions pour les périodes de sécheresse en Chine en 1928-1931, 1936 et 1941, entre un et quatre millions pour les inondations du Yangzi Jiang en 1931). Les tremblements de terre les plus terribles font quant à eux rarement plus de 100000 morts, et les éruptions volcaniques rarement plus de 10000.

À ces terribles chocs, les sociétés apportent différents types de réponses. La première et principale forme de résilience des hommes au sein des sociétés traditionnelles soumises à des stress environnementaux mettant en péril leurs moyens de subsistance consiste à s'installer ailleurs. Cette capacité à pouvoir migrer et recommencer sa vie dans un nouvel environnement est restée une option pendant très longtemps :

«En 1912-1915 encore, par exemple, alors qu’une sécheresse intense affectait le Sahel en Afrique de l'Ouest, des millions de personnes migrèrent vers le sud, une solution encore possible alors que l'Afrique de l'Ouest était huit fois moins peuplée que de nos jours et les contrôles aux frontières inexistants ou inefficaces. [...] pendant la grande majorité de notre histoire, la mobilité était la solution, la seule forme nécessaire de résilience aux catastrophes naturelles. $\$ 55$

Migrer est bien sûr beaucoup moins facile aujourd'hui alors que les frontières de nombreux pays deviennent de plus en plus hermétiques, même si cela a été en partie compensé depuis les années 1950 par le développement de techniques et d'infrastructures permettant d'apporter des secours d'urgence à des

54. Je suis pour cette partie l'excellent chapitre de John McNeill, "Can History Help Us with Global Warming?”, in Campbell K. (ed), Climatic Cataclysm: The Foreign Policy and National Security Implications of Climate Change, Washington, Brookings Institution Press, 2008, pp. 26-48. Sauf indication contraire, les arguments, exemples et citations proviennent de cet article qui est bien sûr bien plus complet que les quelques éléments présentés ici.

55. McNeill, J., "Can History Help Us with Global Warming?”, op. cit., p. 30. 
millions de personnes ou de procéder à des évacuations massives. D’autres formes traditionnelles de résilience, comme l'accroissement de la natalité, peuvent également être mises en œuvre.

John McNeill propose quelques généralisations à propos des effets politiques et sociaux de ces catastrophes naturelles à travers l'histoire. Tout d'abord, les catastrophes ont souvent un effet paradoxal sur les sociétés, attisant les divisions d'une part et encourageant la solidarité de l'autre. Par exemple, aux États-Unis en 2005, l'ouragan Katrina a donné lieu d'un côté à des scènes de pillage et d'horreur ainsi qu'à des débats houleux entre des hommes politiques au sujet de leurs responsabilités respectives, et de l'autre à des élans de générosité et de solidarité envers des victimes en provenance de tout le pays et au-delà. D'une manière plus générale, les conflits sont fréquents pendant ou après des catastrophes naturelles :

\begin{abstract}
«Les sociétés les plus vulnérables peuvent facilement sombrer dans le banditisme, les violences religieuses ou ethniques, ou même la guerre civile dans le cas de sécheresses graves, comme ce fut par exemple le cas en Éthiopie dans les années 1970 et 1980. Les hommes peuvent facilement abandonner leur retenue et un civisme de façade lorsqu'ils s'y sentent contraints par les événements. » 56
\end{abstract}

Par ailleurs, il n'est pas rare de voir les personnes affectées par des catastrophes naturelles s'en prendre à certaines minorités, transformées en boucémissaires, comme ce fut le cas en Europe après la peste noire, où les Juifs furent couramment accusés d'empoisonner les puits. Beaucoup plus récemment, après le tremblement de terre de Kantô en 1923, des Japonais attaquèrent et tuèrent plusieurs milliers de Coréens dans cette région. Les gouvernements modernes, qui sont censés sinon prévenir, du moins répondre rapidement aux catastrophes en organisant les secours, peuvent également être fortement pris à partie et déstabilisés par les répercussions sociales des désordres naturels. Par exemple, les épidémies de choléra en Europe contribuèrent à intensifier les divisions dans la société. Affectant principalement les populations pauvres vivant dans de mauvaises conditions sanitaires, le choléra alimentait les soupçons des classes populaires envers les riches et les gouvernements qu'elles accusaient de collusion et d'empoisonnement. Ces épidémies accentuèrent les tensions et participèrent probablement des agitations révolutionnaires entre 1830 et 187057 . Enfin, les catastrophes naturelles avivent souvent les sentiments religieux, et il n'est pas rare de voir naitre diverses sectes religieuses sur les décombres de pays ou de régions ruinés par des ouragans ou des tsunamis.

56. Ibid., p. 35 .

57. Chevalier L. (ed), Le Choléra : la première épidémie du XIXe siècle, La Roche-sur-Yon, imprimerie centrale de l'Ouest, 1958 ; Evans R., "Epidemics and Revolutions: Cholera in Nineteenth-century Europe”, in Ranger T., Slack P. (eds), Epidemics and Ideas: Essays in the 
Par ailleurs, et dans un tout autre ordre d'idées, puisqu'une réduction drastique de la consommation de combustibles fossiles est nécessaire si l'on veut enrayer le réchauffement continu de l'atmosphère, il est très important d'observer les circonstances dans lesquelles se sont réalisées les précédentes transitions énergétiques ${ }^{58}$. L'histoire des mutations technologiques au cours des siècles passés montre que ces changements peuvent être assez rapides et abrupts mais qu'ils ne se produisent que lorsque les circonstances sont favorables, ce qu'il n'est pas forcément facile de reproduire ou de susciter politiquement. L'histoire offre par ailleurs peu d'espoir quant à la capacité des hommes à réduire volontairement leur consommation d'énergie.

«Il y a très peu d'exemples dans le monde, de sociétés (par opposition aux ermites ou aux moines) renonçant volontairement aux fruits d'un système très énergivore, ou embrassant un niveau de vie inférieur, comme le supposerait la réduction de consommation d'énergie (à moins de gains d'efficacité). » 59

L'abolition de l'esclavage fait office d'exception à cette règle générale puisque de nombreuses sociétés renoncèrent collectivement, au cours des XIX ${ }^{e}$ et $\mathrm{XX}^{\mathrm{e}}$ siècles, à jouir des bénéfices d'esclaves et de serviteurs qui procuraient les mêmes services que nos «esclaves énergétiques" aujourd'hui 60 . Mais McNeill rappelle que l'abolition de l'esclavage fut de ce point de vue une exception notable qui prit beaucoup de temps, nécessita une guerre civile dans le cas des États-Unis, et fut contingent de l'arrivée des énergies fossiles et de développement géopolitiques bien difficiles à reproduire.

S'appuyant sur le consensus scientifique, McNeill considère enfin qu'il est "prudent, à la fois intellectuellement et pratiquement, d'accepter que l'atmosphère et les océans se réchauffent, comme les indices nous le montrent, et que ces tendances vont s'accélérer dans les décennies à venir » 61 . A l'heure où les climato-sceptiques continuent d'alimenter de virulentes controverses et d'instrumentaliser certains travaux historiques, le conseil de McNeill devrait inciter les historiens à être à la fois plus prudents et plus courageux. Plus prudents, pour ne pas alimenter l'impression que " le climat ayant toujours varié, il n'y a pas lieu de s'inquiéter »; et plus courageux, pour oser étudier les relations entre les hommes et le climat, malgré les accusations de déterminisme qui frappent souvent ceux qui s'y risquent.

Historical Perception of Pestilence, Cambridge, Cambridge University Press, 1992, pp. 149173.

58. Sur l'histoire de l'énergie, voir en particulier les travaux de Vaclav Smil, par exemple : Energy in World History, Bolder: Westview, 1995, ainsi que Crosby A. W., Children of the Sun: A History of Humanity's Unappeasable Appetite for Energy, New York, W.W. Norton, 2006.

59. McNeill, J., "Can History Help Us with Global Warming?", op. cit., p. 42.

60. Mouhot J.-F., Des Esclaves énergétiques. Réflexions sur le changement climatique, Seyssel, Champ Vallon, 2011.

61. McNeill, J., "Can History Help Us with Global Warming?”, op. cit., p. 26. 


\section{Deux défis pour les historiens}

\section{Le climat a toujours changé...}

Il existe une posture largement répandue selon laquelle le climat ayant toujours fluctué, le réchauffement actuel ne serait qu'un nouvel avatar d'une histoire déjà ancienne, et qu'il n'y a par conséquent pas lieu de sombrer dans le catastrophisme. Toute aussi courante est la position qui affirme qu'il y a toujours eu des catastrophes et des prophètes de l'Apocalypse, quand celle-ci ne s'est jamais produite ${ }^{62}$. Bien sûr, ces opinions sont rarement exprimées en des termes aussi explicites et caricaturaux, mais elles n'en sont pas moins bien présentes en filigrane chez certains historiens. Elles trouvent parfois leur origine dans une volonté manifeste de l'historien de " rassurer », ou paradoxalement peut-être dans un désir de ces mêmes historiens de contribuer aux débats d'idées sur le changement climatique (où ils se sentent parfois marginalisés) grâce à des arguments nouveaux ou provocateurs. Quelles que soient les raisons, étant donnés les enjeux du débat climatique, il est important que les historiens soient attentifs à ne pas semer davantage la confusion en veillant à ne pas sortir de leur rôle d'historien, en s'abstenant d'intervenir dans des domaines dans lesquels ils ne sont pas compétents. Laissons de côté les historiens insinuant que la thèse "réchauffiste » est un complot, comme Pascal Acot 63 et prenons l'exemple d'un livre récemment publié par Emmanuel Garnier ${ }^{64}$, l'un des très rares livres de climatologie historique en français avec ceux d'Emmanuel Le Roy Ladurie. L'auteur se défend de nier le réchauffement climatique anthropique 65 mais laisse entendre que les certitudes scientifiques sur certains points sont remises en cause par les recherches, apparemment plus précises (!), des historiens. Par exemple il estime que les thèses soutenues par quelques «scientifiques de renom » affirmant que le réchauffement des trente dernières années est « directement imputable à l'intensification de l'activité solaire 66 » sont «parfois solides ». Message pour le moins ambigu d'un historien à l'heure où le consensus scientifique a clairement discrédité l'influence de l'activité solaire dans le réchauffement actuel ${ }^{67}$.

D’autres passages du livre posent problème. Par exemple, la quatrième de couverture :

62. C'est le type d'arguments employés par Walter F., Catastrophes: Une histoire culturelle XVIeXXIe siècles, Paris, Le Seuil, 2008, et plus récemment par Bruckner P., Le Fanatisme de l'Apocalypse, Paris, Grasset, 2011.

63. Voir le compte-rendu du livre par Godard O., «À propos du livre de Pascal Acot, Climat, un débat dévoyé ? ", Natures Sciences Sociétés, n¹9, 2011, p. 282-286.

64. E. Garnier, Les Dérangements du temps : 500 ans de chaud et de froid en Europe, Paris, Plon, 2010.

65. Ibid., pp. 102-103.

66. Ibid., p. 63.

67. Voir par exemple le rapport de l'Académie des Sciences, Le changement climatique, 16 octobre 2010, p. 5 (http://www.academie-sciences.fr/activite/rapport/rapport261010.pdf, consulté le 13/11/2012). 
"La certitude qu'ont nos contemporains de vivre un "changement" climatique ne date pas d'aujourd'hui. Tiré d'archives inédites, le présent ouvrage souhaite offrir une autre réalité des fluctuations climatiques $[\ldots]$. La première constatation concerne le caractère neuf du réchauffement observé depuis 30 ans. La réponse de l'historien est sans appel. Bien avant le Global Warning [sic], nos ancêtres connurent des épisodes parfois très chauds dont l'intensité fit reculer les glaciers alpins à des niveaux inférieurs à ceux d'aujourd'hui. »

Le fait est que le livre, attaqué pour la faiblesse de son appareil critique par Christian Pfister dans une recension récente ${ }^{68}$, n'apporte aucune preuve de ces allégations. Garnier laisse entendre que le caractère nouveau du réchauffement climatique global est mis à mal par ses recherches alors qu'il présente des résultats qui ne concernent que le bassin méditerranéen. Qu'il ait pu y avoir, pendant une courte période après 1676, un réchauffement localisé en Méditerranée équivalent au réchauffement actuel ne remet pas en cause les conclusions du GIEC 69. Encore faudrait-il qu'Emmanuel Garnier prouve la réalité de ce réchauffement. Or le graphique incomplet qu'il présente à l'appui de sa démonstration ne prouve rien ${ }^{70}$.

Plus généralement, l'idée que, le climat ayant toujours varié, il n’y a pas lieu de s'inquiéter est une tendance naturelle. Mais les prémisses de ce raisonnement sont problématiques. Si l'homme en tant qu'espèce a remarquablement prospéré jusqu'à aujourd'hui en dépit des variations du climat, pour les sociétés prises individuellement, c'est une autre histoire, comme nous l'avons vu ci-dessus. Ensuite, répétons-le, le réchauffement climatique annoncé n’a pas d'équivalent dans l'histoire des civilisations. Par conséquent, si les historiens ne veulent pas obscurcir la compréhension de leurs lecteurs ou courir le risque de les rassurer de manière trompeuse, ils devraient veiller à équilibrer leur récit en rappelant en quoi le passé est un mauvais guide du futur en la matière et ne pas oublier leurs responsabilités : des séismologues italiens ont été condamnés en octobre 2012 à six ans de prison pour « homicide par impru-

68. Pfister C., « Garnier E., Les dérangements du temps. 500 ans de chaud et de froid en Europe », Annales, Histoire, Sciences Sociales, nº6, janvier-mars 2011, p. 303.

69. E. Garnier n'est pas le seul chercheur à commettre ce type d'amalgame. Dans son livre récent (Climate and Catastrophe in Cuba and the Atlantic World in the Age of Revolution, Durham: University of North Carolina Press, 2011), Sherry Johnson écrit que « la phase actuelle de stress climatique n'est pas unique » et qu' "un cycle similaire [de réchauffement] - une anomalie ayant duré cinquante ans - se produisit à la fin du XVIIIe siècle ». Or l'auteur ne donne aucun élément valable pour justifier cette affirmation étonnante, et omet de préciser que son étude ne s'intéresse qu'aux Antilles. Voir mon compte-rendu du livre à paraître dans Environmental History en 2013.

70. Voir pp. 64-65 du livre et Fig. 4 : «Anomalies des températures annuelles dans le bassin méditerranéen entre 1655 et 2007 ", section centrale du livre, non paginée. Si ce graphique montre effectivement un bref moment assez chaud à la fin du XVII siècle, ce moment n'est pas aussi long dans le temps que le réchauffement actuel et on ne voit pas non plus comment l'auteur peut calculer une moyenne mobile sur 11 ans avec une lacune dans les données de quatre ou cinq ans au moins. 
dence » parce qu'ils avaient sous-estimé les risques de tremblement de terre à L'Aquila (Italie) qui fit plus de 300 victimes en 2009. On peut ne pas approuver cette décision judiciaire tout en comprenant la colère des habitants de cette localité des Abruzzes à qui les séismologues avaient recommandé, une semaine avant le tremblement de terre et malgré des signes inquiétants, de « dormir tranquilles » et de boire « tranquillement un verre de Montepulciano », le vin local, plutôt que de s'inquiéter ${ }^{71}$.

\section{La question du déterminisme climatique}

Vouloir rassurer les gens ou relativiser la menace climatique comme le fait Emmanuel Garnier peut partir d'une bonne intention. Il y a en effet un risque réel que le catastrophisme soit contre-productif en donnant l'impression que le futur est déjà écrit, et que l'homme ou les sociétés n'ont plus aucune marge de manœuvre ${ }^{72}$. La peur peut conduire au découragement et à l'apathie. Mais ce risque affecte principalement la partie de l'opinion qui est convaincue par le consensus scientifique. Par ailleurs, la peur peut aussi conduire à un sursaut salutaire : de manière convaincante, Jean-Pierre Dupuy fait ainsi d'une posture catastrophiste la seule issue possible afin d'éviter la catastrophe ${ }^{73}$. Pour ceux qui, par l'effet des médias, ne savent que croire, un excès de relativisme justifie l'insouciance et l'inaction, ce que les lobbies de l'énergie ont parfaitement bien compris.

Pour y voir plus clair, il serait utile de disposer d'études fines analysant les effets du climat sur l'homme. Or les soupçons de déterminisme qui pleuvent sur les chercheurs dès qu'ils se hasardent à étudier les relations passées entre hommes et climat ou à faire des conjectures sur leurs relations futures inhibent les recherches en ce domaine. «Déterministe » est devenu une sorte d'insulte, utilisée pour attaquer les travaux de collègues. La connotation négative du terme a une origine historique. Loin de disparaître après Montesquieu, la théorie des climats, même si elle recula largement, continua à survivre sous différentes formes jusqu'au Xx ${ }^{\mathrm{e}}$ siècle (Montesquieu lui-même avait déjà été accusé d'une forme de déterminisme climatique). Cette idée fut largement reprise par plusieurs chercheurs, en particulier par les géographes Friedrich Ratzel, Ellen Semple et Ellsworth Huntington, un professeur américain dont les travaux controversés suscitèrent un grand intérêt parmi le public au début du XXe siècle.

Pendant l'époque coloniale, les théories expliquant la pauvreté des régions tropicales (ou la soi-disant paresse des autochtones) par le climat étaient

71. Ridet P., «La responsabilité des scientifiques mise en cause dans le drame de L'Aquila », Le Monde, 4 juin 2010.

72. O’Neill S., Nicholson-Cole S., “'Fear Won't Do It': Promoting Positive Engagement With Climate Change'”, Science Communication, vol. 30, n³, 2009, p. 355.

73. Dupuy J-P., Pour un catastrophisme éclairé: Quand l'impossible est certain, Paris, Seuil, 2002. 
populaires et commodes. Des variantes racistes ne tardèrent pas à se développer à partir de ces explications par le climat. Ainsi, certains soutenaient que les habitants des zones tropicales avaient dégénéré en des « races » paresseuses. Ces théories justifiaient la colonisation et l'intervention des Occidentaux appelés à enseigner aux autochtones le travail et la discipline. Huntington entreprit de classer les civilisations suivant leur climat. Ralph Waldo Emerson, un progressiste, déclarait : « là où poussent les bananes, l'homme est sensuel et cruel ». On comprend qu'avec de telles dérives, ce type d'idées - et la géographie en général - aient été fortement discrédités après 1945.

Pourtant, on peut regretter l'amalgame souvent entretenu entre le point de départ de nombreuses études anciennes (« la plupart des pays pauvres ou sous-développés du monde sont situés dans la zone intertropicale ») et les dérapages évoqués plus haut. Or ces dérives racistes n’avaient rien d'inévitable. Des universitaires du III Reich furent ainsi envoyés au Brésil dans les années 1930 pour enquêter sur l'adaptation au climat tropical d'Allemands émigrés depuis trois générations, espérant montrer que les Aryens pouvaient prospérer n'importe où. Mais ils trouvèrent la troisième génération d'émigrants allemands aussi misérables que leurs voisins métis, et durent renoncer à publier leurs résultats ${ }^{74}$. Pourtant, le fait que le sous-développement de pays de la zone tropicale puisse en partie s'expliquer par le climat (qui rend ces pays moins susceptibles de réussir économiquement du fait notamment de problèmes sanitaires) décrédibilise ceux qui l'imputent à des facteurs « culturels ».

L'accusation de déterminisme plane sur quiconque ose attribuer un rôle jugé trop important au climat ou à l'environnement. Le Roy Ladurie a eu une influence considérable en ce domaine. Craignant lui-même que sa carrière n'en pâtisse, il se refusait à étudier les rapports entre hommes et climats et écrivait ainsi que «les conséquences humaines [de la longue durée climatique] semblent assez faibles, peut-être négligeables, à coup sûr difficiles à déceler 75 ». Le spectre du déterminisme continue à inhiber ce genre d'études.

Le débat a récemment resurgi à propos de ceux qui tentent de mesurer les impacts futurs du réchauffement climatique. Selon certains observateurs, le déterminisme climatique serait de retour de manière plus ou moins insidieuse aujourd'hui. Le déterminisme est un danger réel, mais l'obsession de certains collègues à débusquer ce travers partout l'est également. Ceux qui se risquent, par exemple, à tenter de chiffrer le nombre potentiel de réfugiés climatiques sont attaqués sous le prétexte que leurs modèles ne prendraient pas suffisamment en compte la faculté d'adaptation des sociétés. Par crainte de passer pour déterministes, beaucoup d'auteurs ont préféré ignorer entièrement le rôle $\mathrm{du}$

74. Dean W., With Broadax and Firebrand: The Destruction of the Brazilian Atlantic Forest, Berkeley, University of California Press, 1995, pp. 243-244.

75. Le Roy Ladurie E., Histoire du climat depuis l'an mil, op. cit., p. 92 ; son opinion a considérablement évolué depuis. 
climat dans les affaires humaines. De ce point de vue, la voie « possibiliste » proposée par Paul Vidal de la Blache ou Lucien Febvre ${ }^{76}$ - que l'on pourrait résumer par "la nature propose, l'homme dispose »-n'a guère été suivie d'effets et a plutôt conduit à une abdication devant le problème des relations entre les hommes et leur climat, « une fuite élégante devant les responsabilités », écrit Georges Bertrand : «Le possibilisme tel qu'on le pratique n'est plus pour l'historien ou le géographe qu'une façon d'éluder le problème des relations entre les sociétés humaines et les milieux dits naturels 77 ».

S'il est donc important de garder en mémoire que le futur n'est pas prédéterminé et d'être attentif à la tendance auto-réalisatrice des prophéties, il faut espérer que les historiens sauront se dégager des inhibitions engendrées par les excès des déterministes du siècle passé et recommenceront à étudier sereinement les relations entre les hommes et le climat. De tels travaux sont importants car, malgré leurs limites, les études d'impact peuvent apporter un éclairage utile dans les discussions contemporaines. Elles permettent de mieux appréhender le problème du réchauffement climatique global actuel par rapport à la simple projection de hausses de températures, qui mobilisent moins l'opinion (envisager une augmentation de température de $3^{\circ} \mathrm{C}$ en 2100 reste plus abstrait que d'imaginer, par exemple, la possibilité de plusieurs dizaines ou centaines de millions de réfugiés climatiques).

L'histoire peut donc nous aider de diverses manières : en nous montrant comment les hommes ont été affectés et ont réagi à diverses catastrophes naturelles (en particulier climatiques) dans le passé ; en s'intéressant aux interactions entre l'homme et le climat ; mais aussi, plus généralement, en revisitant de très nombreuses questions historiques traditionnelles à l'aune de notre nouvelle fragilité climatique et énergétique (la liste des questions à reconsidérer est quasiment infinie). Il faut espérer qu'un plus grand nombre d'historiens prendront rapidement la mesure des problèmes énergétiques et climatiques actuels et qu'ils seront à la fois prudents et courageux pour oser explorer avec les outils modernes des territoires trop longtemps considérés avec suspicion. C'est à ce prix que la recherche pourra avancer. Il faudra pour ce faire que les historiens apprennent à travailler de manière toujours plus interdisciplinaire, afin de tirer profit des travaux des archéologues, paléontologues, climatologues, économistes, philosophes et d'autres qui partagent avec eux le même terrain d'étude.

76. Massard-Guilbaud, G. «Historiens et géographes français et la relation de l'homme au milieu et à l'environnement, de Vidal de la Blache aux programmes de recherche interdisciplinaires de la fin du XXe siècle ", in Étudier les interactions hommes-milieux. Questions et réflexions autour des pratiques de la recherche scientifique en environnement, édité par Samuel Robert: Versailles, Quae, 2013.

77. Bertrand G., « Pour une histoire écologique de la France rurale », in Duby G., Wallon A. (ed), Histoire de la France rurale, tome 1, Paris, Seuil, 1975. 